\title{
Respondenz Abschnitt 3: Geschichte ist (k)ein Dosenöffner
}

Geschichte ist kein Dosenöffner! Dachte ich. Und so wollte ich eigentlich auch meine Respondenz beginnen. Doch dann waren da diese drei Texte, auf die ich zu respondieren hatte, und plötzlich war ich mir nicht mehr so sicher. Zunächst betrieb Christoph Borbach ${ }^{1}$ epistemologisches Reverse Engineering und zerlegte aus der Ferne das Behm'sche Echolot in seine Einzelteile. Dann schlich sich Michael Ott mittels Rekonstruktionen in eine Druckerwerkstatt der frühen Neuzeit, um verborgenen Praktiken in einem sonst verschlossenen Raum auf die Spur zu kommen. Und schließlich spannte Oliver Schlaudt einen ganz weiten Bogen vom Archaeopteryx bis in unsere Zeit und versäumte es nicht, - Thema Zweckentfremdung - noch kurzerhand Leichen, beschwert mit Verbrennungsmotoren, in einem Gewässer zu versenken. Aber der Reihe nach.

\section{Un-Boxing History - dosenöffnende Geschichte}

Geschichte öffnet Black Boxes. Sie bringt Licht ins Dunkel der Vergangenheit und erhellt uns, was vergessen wurde, was vielleicht an Tradition und Überrest noch vorliegt, jedoch nicht mehr selbsterklärend bzw. verständlich ist. Legt man einen sehr weiten Begriff von Black Box an, der den in diesem Band verwendeten Begriff noch ein wenig überdehnt, so ist jede Form der historischen Arbeit ein Öffnen einer Black Box, einer raum-zeitlichen Unbekannten (oder unbekannten Raum-Zeit?), die uns heute fremd und opak geworden ist. Nicht ganz so weit dehnen muss man den BlackBox-Begriff für technikhistorische Unternehmungen, die (technische) Artefakte in den Blick nehmen. Hierbei ist es einerlei, ob es sich dabei um eine Darstellung vergangener Technik handelt, die heute nicht mehr verwendet wird bzw. nicht mehr existent ist, oder um gegenwärtige technische Objekte, deren Geschichte erzählt werden soll. In beiden Fällen ist es Ziel, mindestens unter anderem die Funktionsweise des Artefakts zu klären. Dabei wird die Black Box geöffnet.

Jedoch - und nun komme ich zum Dosenöffner - geschieht dies nicht indem man das Verschlossene wieder versucht aufzuhebeln, nachdem es versiegelt wurde. Vielmehr versuchen Historiker`innen an den Anfang des Ganzen zu springen - nicht in

\footnotetext{
1 Zusammenfassungen, Verweise und Zitate der Vorträge, die in dieser Respondenz besprochenen werden, beziehen sich sämtlich auf die jeweiligen Vortragsmanuskripte und werden im Folgenden nicht eigens nachgewiesen. Vgl. die jeweiligen Druckfassungen in diesem Band.
} 
einer Zeitmaschine, sondern unter Zuhilfenahme von Quellen und historischer Methode - und die Geschichte des Werdens und damit auch des Versiegelns zu erzählen. Rollt man die Geschichte eines Artefakts, das uns heute nur noch als Black Box bekannt ist, von dieser Seite her auf und erkennt und beschreibt es, noch bevor es versiegelt wurde, so ist ein nachträgliches Un-Boxing nach dem Versiegeln gar nicht nötig. Beobachtet man die Black Box vor dem und beim Verschließen, so muss sie nicht im Nachhinein aufgehebelt werden. Technikgeschichte muss, so praktiziert, also gar kein Dosenöffner sein, der gewaltsam einen Deckel lüftet. Auf diese Weise macht Geschichte den Inhalt von Black Boxes zwar zugänglich, auf eine gewisse Weise öffnet sie sie auch, aber ohne sie nach dem Verschließen aufzubrechen.

Geschichte ist also kein Dosenöffner. Soweit war ich eingangs im Hinblick auf Black Boxes. Doch dann kamen die Beiträge der Sektion „Welt, Werkzeug und Wissen“ und erinnerten mich daran, dass es auch anders geht. Hier wird nämlich gleich drei Mal ein - je etwas unterschiedlich gelagerter - historischer Zugang gewählt, um Black Boxes von hinten her aufzubrechen. Nicht das Verschließen wird hier begleitet, sondern es wird auf drei verschiedene Weisen versucht, die Black Box nachträglich aufzubrechen. Es wird also ein Dosenöffner angesetzt, um an den verborgenen Inhalt der Schachtel zu gelangen. Ein medienhistorischer Ansatz würde dazu, so Borbach, von einem „materiale[n] Ausgangspunkt“ eine „in die Zukunft schreitende [...]“ Geschichte erzählen. Ich würde mit dem oben Beschriebenen für die (Technik)Geschichte präzisieren wollen, dass der Historiker seine Untersuchung vor diesem materialen Ausgangspunkt beginnt und dann die Geschichte - oder (Medien)Geschichte, wie Borbach es fasst - von dort aus weitererzählt. Sein Ansatz des epistemologischen Reverse Engineering will es anders machen, er schreitet zurück und geht von einem „Endpunkt [aus], der auf seine Vorgeschichte“ hin untersucht werden soll. Ähnlich zu verstehen ist das historische Vorgehen, auf das Michael Ott sich in seiner Darstellung des frühen Buchdrucks mit beweglichen Lettern bezieht. Auch hier wird zur Öffnung der Black Box ex post von überlieferten Artefakten und Berichten auf frühere Praktiken rückgeschlossen. Es geht in beiden Fällen um eine Rekonstruktion, die zu erhellen versucht, wie die Black Box jeweils in ihrem Innern funktioniert(e). Borbach, der mit Simondon dafür argumentiert, verschlossene Technik verbaliter aufzuschrauben, um ihre „Prozessarchitektur“ zu erkunden, muss sich im Falle des von ihm gewählten „Behmlots Type 1“ jedoch aufgrund der Unverfügbarkeit des Objekts mit Fotos und der Patentschrift zufriedengeben. Im Falle der von Ott betrachteten Gutenberg'schen „Laborwerkstatt“ ist das Untersuchungsobjekt gänzlich abwesend und nur über einzelne materielle oder textuelle Zeugnisse zugänglich.

Noch einmal anders verhält es sich bei Oliver Schlaudt. Er vergleicht die Zweckentfremdung - „Exaptation“ - die Menschen schon immer vornahmen, um sich beispielsweise Naturgegenstände wie Stoßzähne, Steine, Holzstücke, etc. anzueignen, mit unserer heutigen Aneignungspraxis. Oder vielmehr: Mit dem uns verbleibenden Handlungsraum - marge de manœvre - für diese Form der Aneignung durch Zweckentfremdung bei modernen technischen Artefakten. Die Schwierigkeit bei dem 
Versuch, als Black Box verstandene Naturgegenstände zu öffnen, besteht darin, dass das klassische Un-Boxing hier nicht funktioniert. Zwar kann man Naturgegenstände aufbrechen, jedoch kommt nur wieder das zum Vorschein, was auch schon an der Oberfläche zu sehen war. Die möglichen Funktionen, die ein solcher Gegenstand in sich birgt, bleiben verborgen. Möchte man an diese Black Box ran, muss man es auf andere Weise versuchen: Mittels Exaptation erkunden die Menschen die „Zweckmäßigkeiten“ dieser Black Boxes und das immer wieder aufs Neue in immer weiteren Neukombinationen. In diesem Fall haben wir es sozusagen mit einem zahnlosen Dosenöffner zu tun, der die Black Box als gegeben hinnimmt und - über eigenen kreativen Umgang damit - das Beste daraus macht. Dies hat auch heute noch häufig einen improvisatorischen Stil, wenn der Aschenbecher als Briefbeschwerer dienen muss oder ein Topf als Trommel. Dennoch möchte Schlaudt diesen Werkzeuggebrauch nicht rein als Suche des Zwecks nach geeigneten Mitteln verstanden wissen, „sondern das gegebene Mittel ,drängt` zu neuen Zwecken.“ So verstanden, stellen diese Exaptationen - nach Ansicht von Archäologen und Anthropologen - kulturellen Fortschritt dar. Schlaudt überträgt diese Erkenntnisse auf das Beispiel nicht-funktionierender Technik. Er führt hier in den Worten des Philosophen Alfred Sohn-Rethel das Beispiel des technikwiderständigen Neapel des frühen 20. Jahrhunderts an, in dem Technik prinzipiell kaputt sei, damit aber - für den findigen, exaptationsfähigen Neapolitaner - erst zu funktionieren beginne. Die bei Schlaudt über fünf Thesen hinweg entwickelten Gedanken münden in eine Schicksalsfrage, ob - in einem von ihm nur für gedankenexperimentelle Zwecke geteilten deterministischen Technikverständnis à la Simondon - mit der modernen Technik nicht immer weniger Handlungsraum für Zweckentfremdung bliebe. Und schließlich gar keine mehr. Schlaudt deutet nur eine entwarnende Antwort auf diese Frage an. Sollten daran noch Zweifel bleiben, lassen diese sich aber mit einem Blick in die Technikgeschichte zerstreuen: Der Handlungsspielraum blieb bisher zumindest immer erhalten und scheint auch gegenwärtig keiner Verengung entgegenzugehen. Jedoch muss man sich heute vielleicht etwas weniger zufällig-improvisatorisch der Zweckentfremdung nähern, als das bei Schlaudt beschrieben ist. Vielleicht muss man sich eher gezielt-subversiv um Exaptation bemühen. Dies ist auch, was sich mit der Technikgeschichte zeigen lässt. Für die Geschichte modernen Technikumgangs, insbesondere den mit Mobilitätsmaschinen, spricht Kurt Möser explizit von „subversiven Verwendungsmöglichkeiten“, ${ }^{2}$ die sich ganz bewusst gegen die üblichen und intendierten Nutzungsformen stellen.

2 Möser 2018. 


\section{Mensch und Technik}

Die von Schlaudt angeführte, technikdeterministisch inspirierte Schicksalsfrage bringt uns - weg von der Geschichte als Dosenöffner - hin zu der Frage nach der Bedeutung bzw. nach der Rolle des Menschen im Wechselspiel mit der Technik. Schlaudt sieht, dann doch in Opposition zu diesem Determinismus, den Menschen in der Verantwortung, sich gegen das Verlorengehen der marge de manœvre zu wehren. Er führt dafür niemand geringeren als den im Kontext der Black Boxes (und im Rahmen unseres Workshops besonders gerne) aufgerufenen Vilém Flusser ins Feld, wenn er unterstreicht, dass dieser „das Abwürgen der Exaptation [...] als Kontrollgewinn“ interpretiere. Die Reflexion des Spannungsverhältnisses Mensch-Technik ist überhaupt - neben dem allgegenwärtigen Arbeitsbeispiel der Black Boxes - das heimliche verbindende Element. So befindet sich bei Borbach der Mensch in „Maschinenparks“, in embedded systems, die er öffnen muss, um zu sehen, wie es um diese steht. Und - ich ergänze - zur Selbsterkenntnis in dieser Verflochtenheit. Wenn er dazu epistemisches Reverse Engineering betreibt, dann ist die Black Box - in seinen Worten das „Gehäuse“ - das er öffnet, nicht nur eine Grenzfläche zwischen innen und außen, sondern zugleich ein Interface, das unsere Seh- und Bediengewohnheiten beeinflusst. Dieses Interface vermittelt zwischen Mensch und Maschine und muss für das Un-Boxing (im Dosenöffnungsverfahren) aufgebrochen werden. Auch Otts Erkenntnisinteresse entspringt dem Wunsch, das gegenwärtige „Mensch-MaschineArrangement“ im Spannungsfeld zwischen Buchdruck und Textverarbeitung zu verstehen. Zielobjekt seines Un-Boxing ist die neuzeitliche Buchdruckerwerkstatt mit ihren Praktiken; sie ist für ihn Folie für den Vergleich mit den heute gängigen Praktiken der Textverarbeitung. Spielte im Buchdruck die Aufgabenteilung noch eine entscheidende Rolle - von Autor, über Lektor, bis hin zum Buchhändler hatte jeder seine definierten und voneinander abgegrenzten Aufgaben - so ist in der Textverarbeitung jeder selbst seines Glückes Schmied. In der modernen Produktion von Textströmen können die verschiedenen Aufgaben, dank Textverarbeitungsprogrammen, im Aufgabenbereich einer einzelnen Person zusammenfallen. Gleichzeitig ist es, so Ott, genau diese Software, die uns vor „den Fluten des Textes“ bewahrt, da in ihr zahlreiche Eigenschaften der früheren Buchdruckkultur eingeschrieben und persistent sind. Somit wird „Microsofts ,Word“ [...] die letzte Bastion der Druckkultur, weil diese als Matrix tief in der Software und deren Benutzerschnittstelle integriert ist. " Bis also die Algorithmen einmal vollständig die Texterstellung und -verarbeitung übernehmen ist ein kulturpessimistischer Abgesang verfrüht. Dann jedoch wäre es, so möchte ich anmerken, an der Zeit für ein erneutes Un-Boxing, diesmal der textgenerierenden Algorithmen. Hierfür wird ein historisches Dosenöffnen wohl nicht den gewünschten Zweck erfüllen. Wie Algorithmen sich als Black Box öffnen lassen ist Gegenstand anderer Beiträge in diesem Band. 


\section{TechnikGeschichteTheorie}

Wie auch immer es sich mit dem Öffnen von Algorithmen verhalten mag, Geschichte ist wohl doch ein Dosenöffner. Die drei Beiträge in dieser Sektion haben eindrücklich gezeigt, wie man auch vom Endpunkt her, wie Borbach es beschreibt, Black Boxes ihre Geheimnisse entlocken kann. Dabei argumentieren alle drei Ansätze historisch, jeder jedoch auf seine Weise. Haben Ott und Borbach mit der Druckerwerkstatt und dem Echolot historische Black Boxes geöffnet, so zeigt Schlaudt, wie Menschen historisch die „schraubenlose Black Box“ öffneten, die sie in Naturgegenständen fanden. Die untersuchten Black Boxes sind von ganz unterschiedlicher Art. Dies entspricht nicht nur dem Zugang des interdisziplinär und explorativ angelegten Workshops, sondern auch dem weiten Black-Box-Begriff wie er in der (Technik)Geschichte praktiziert wird. Diese weite Definition erlaubt es, beim Öffnen von verschiedensten Black Boxes die Zusammenhänge außer-technischer und technischer Aspekte herauszustellen und somit eine Kontextualisierung vorzunehmen. Der Technikgeschichte stünde es dabei gut zu Gesicht an die zu untersuchenden Gegenstände auch wirklich Hand anzulegen und die zu erforschenden Artefakte tatsächlich auseinanderzunehmen - ähnlich wie Simondon das praktiziert. Damit nicht genug, es liegt nahe, an dieser Stelle technisches Sachverständnis, wenn schon nicht einzufordern, so doch zumindest zu wünschen. Möchte man nämlich nicht nur rein phänomenologisch das zerlegte Artefakt betrachten, sondern tatsächlich die Black Box in ihrem Innersten verstehen, so ist dieses Wissen kaum umgänglich. Deshalb sei hier für ein wahrlich interdisziplinäres Herangehen plädiert, das Technik- und Kulturwissenschaften, Geistes- und Naturwissenschaften zusammenbringt. Erst durch den gemeinsamen Austausch und das voneinander Lernen wird das durch das Un-Boxing ermöglichte Draufschauen auch ein Reinschauen und vielleicht sogar ein Durchschauen der Black Box.

\section{Black Box Technikdeterminismus - ein persönliches Fazit}

Ein abschließendes Wort sei noch zu dem - insbesondere mit Simondon - besprochenen Technikdeterminismus gesagt, mit dem ich ein persönliches Fazit verbinden will. Wie sehr einen akademische Lehrer und das wissenschaftliche Umfeld prägen, in dem man sich bewegt, wird häufig erst bewusst, wenn man einmal mit ganz anderen Mindsets konfrontiert wird. Die bei unserem Workshop und in einzelnen Beiträgen vorgetragene Begeisterung für technikdeterministische Ansätze, vermag ich nicht zu teilen. Gerne folge ich Schlaudt, der diesen Ansatz verwendet, um ihn dann wieder zu verwerfen; darüber hinaus befremdet er mich jedoch. Dies soll nicht heißen, dass mir bis dato jeglicher Technikdeterminismus fremd gewesen wäre - im Umgang mit Vertretern technischer Fachrichtungen trifft man ihn gar häufig an. Die von in 
der Technik- und Wissenschaftsforschung aktiven Geistes- und Kulturwissenschaftlern vorgebrachte Determinismusbegeisterung à la Simondon war mir jedoch in dieser lebendigen Form neu. Es war also in gewisser Weise das Öffnen einer Black Box für mich. Meine Auseinandersetzung damit war instruktiv, wenn auch nicht überzeugend. Ich kann also für mich getrost die Black Box Technikdeterminismus, die auf dem Workshop geöffnet wurde, wieder zumachen. Dass ich einem sozialkonstruktivistischen Ansatz näherstehe, als einem technikdeterministischen war mir schon vor unserem Workshop klar. In welchem Maße dies der Fall ist, ist mir erst jetzt bewusst. Lehrreich war es allemal. So kann ich aber dem Technikdeterminismus an dieser Stelle eine klare Absage erteilen und damit ein kulturpessimistisches Fazit vermeiden. Es ist wichtig, Black Boxes zu öffnen und sich mit ihrem Inhalt zu beschäftigen und nicht von diesem abschrecken zu lassen. Gerade durch historisches Un-Boxing lernen wir viel über unsere (heutige) Welt. Auch und gerade, dass Technik eben nicht deterministisch ist. In diesem Sinne: Ran an die Dosenöffner!

\section{Literaturverzeichnis}

Borbach, Christoph (2020), „Epistemologisches Reverse Engineering oder Über Techniktheorie(n), Gilbert Simondon und das Echolot“, in diesem Band.

Möser, Kurt (2018): Bemerkungen zur Technikgeschichte imaginärer Dinge (Diskussionspapiere | Institut für Technikzukünfte 6), Karlsruhe, https://publikationen.bibliothek.kit.edu/1000083099 (DOI: 10.5445/IR/1000083099).

Ott, Michael (2020), „Black Box Buchdruck“, in diesem Band.

Schlaudt, Oliver (2020), „Zweckentfremdung als Mittelaneignung. Fünf Thesen und eine Schicksalsfrage“, in diesem Band. 